\title{
On a family of contractive maps
}

\section{Milan R. Tasković}

The object of this paper is to study common fixed points of mappings of a complete metric space into itself. The results obtained are generalizations of theorems of Iséki and Ray. An example is given to show that our results are indeed extensions of the theorem of Iséki.

\section{Introduction}

Let $(X, \rho)$ be a metric space. A mapping $T: X \rightarrow X$ is called an $f$-contraction iff for every $x, y \in X$, there exist real numbers $\alpha_{i}=\alpha_{i}(x, y) \quad(i=1, \ldots, 5)$ such that

$$
\begin{aligned}
\rho[T x, T y] \leq & f\left(\alpha_{1} \rho[x, y], \alpha_{2} \rho[x, T x], \alpha_{3} \rho[y, T y], \alpha_{4} \rho[x, T y], \alpha_{5} \rho[y, T x]\right), \\
& \sup \left\{f\left(\alpha_{1}, \alpha_{2}, \alpha_{3}, \alpha_{4}, \alpha_{5}\right): x, y \in x\right\}=\bar{\lambda} \in[0,1),
\end{aligned}
$$

where the mapping $f: R^{5} \rightarrow R$ is increasing, semihomogeneous [that is to say, for all $\delta \geq 0$ one has $\left.f\left(\delta x_{1}, \delta x_{2}, \ldots, \delta x_{5}\right) \leq \delta f\left(x_{1}, x_{2}, \ldots, x_{5}\right)\right]$ and $g(x)=f\left(\alpha_{1} x, \ldots, \alpha_{5} x^{5}\right)$ continuous in the point $x=1$.

In [6] I proved the following result.

THEOREM T. An f-contraction $T: X \rightarrow X$ on a T-orbitally complete [that is to say, every Cauchy sequence $\left\{T^{n} x \mid n \in N, x \in X\right\}$ converges in $X]$ metric space $X$ has a fixed point, and this is unique when $\bar{\lambda}_{0} \stackrel{\text { def }}{=} \sup \left\{\left(\alpha_{1}, 0,0, \alpha_{4}, \alpha_{5}\right\}: x, y \in X\right\}<1$.

In a recent paper [2] Iséki proved the following:

Received 15 July 1975. 
THEOREM I. Let $X$ be a complete metric space, $T_{n}(n=1,2, \ldots)$ a sequence of mappings of $X$ into itself. Suppose that there are nonnegative numbers $a, b, c$ such that for $x, y \in X$, $\rho\left[T_{i} x, T_{j} y\right] \leq a\left(\rho\left[x, T_{i}(x)\right]+\rho\left[y, T_{j}(y)\right]\right)+$

$$
+b\left(\rho\left[x, T_{j}(y)\right]+\rho\left[y, T_{i}(x)\right]\right)+c \rho[x, y],
$$

where $2 a+2 b+c<1$. Then the sequence of mappings $\left\{T_{n}\right\}$ has a unique common fixed point.

(Let $I$ be an infinite set of indices and let $F=\left\{T_{\lambda}: \lambda \in I\right\}$ be a family of maps which map $X$ into itself. A point $\xi \in X$ is a common fixed point for $F$ iff $\xi=T_{\lambda} \xi$ for each $T_{\lambda} \in F$.)

THEOREM 1. Let $X$ be a complete metric space, $\left\{T_{n}: n \in N\right\}$ a sequence of mappings of $X$ into itself. For $x y \in X$, let there exist real numbers $\alpha_{i}(i=1, \ldots, 5)$ such that

(I) $\rho\left[T_{i} x, T_{j} y\right] \leq f\left(\alpha_{1} \rho[x, y], \alpha_{2} \rho\left[x, T_{i}(x)\right], \alpha_{3} \rho\left[y, T_{j}(y)\right]\right.$,

$$
\begin{array}{r}
\left.3_{2} \alpha_{4} \rho\left[x, T_{j}(y)\right], 3_{5} \rho\left[y, T_{i}(x)\right]\right), \\
\sup \left\{f\left(\alpha_{1}, \alpha_{2}, \alpha_{3}, \alpha_{4}, \alpha_{5}\right\}: x, y \in X\right\}=\delta \in[0,1),
\end{array}
$$

where the mapping $f: R^{5} \rightarrow R$ is increasing, semihomogeneous and $g(x)=f\left(\alpha_{1} x, \ldots, \alpha_{5} x^{5}\right)$ is continuous in the point $x=1$. Then the sequence of mappings $\left\{T_{n}\right\}$ has a common fixed point $\xi \in X$, and this is unique when $\sup \left\{f\left(\alpha_{1}, 0,0, \frac{1}{2} \alpha_{4}, \frac{1}{2} \alpha_{5}\right): x y \in X\right\}=\delta_{0}<1$, and for arbitrary $x_{0} \in X$ the sequence

$$
x_{n}=T_{n}\left(x_{n-1}\right), \quad(n=1,2, \ldots),
$$

converges to $\xi$.

Proof. We prove that (2) is a Cauchy sequence, where $x_{0} \in X$ is arbitrary. By assertion (1) we have 
Contractive maps

303

$$
\begin{aligned}
\rho\left[x_{n}, x_{n+1}\right]=\rho\left[T_{n}\left(x_{n-1}\right), T_{n+1}\left(x_{n}\right)\right] \leq f\left(\alpha_{1} \rho\left[x_{n-1}, x_{n}\right], \alpha_{2} \rho\left[x_{n-1}, x_{n}\right],\right. \\
\\
\left.\alpha_{3} \rho\left[x_{n}, x_{n+1}\right], \frac{1}{2} \alpha_{4} \rho\left[x_{n-1}, x_{n+1}\right], \frac{1}{2} \alpha_{5} \rho\left[x_{n}, x_{n}\right]\right) .
\end{aligned}
$$

Now we use the following lemma, proved in [5], p. 198.

LEMMA. Let $f: R^{k+2} \rightarrow R \quad(k \in N)$ be a monotonically increasing (with respect to every real argument) and semihomogeneous mapping, let $g(x)=f\left(a_{0}, a_{1} x, \ldots, a_{k+1} x^{k+1}\right)$ be continuous in the point $x=1$, and let the sequence $\left(x_{n}\right)$ of nonnegative real numbers satisfy the condition

$$
x_{n+k} \leq f\left(a_{0} x_{n}, a_{1} x_{n+1}, \ldots, a_{k} x_{n+k}, a_{k+1} C\right), n=1,2, \ldots,
$$

where $k$ is a fixed natural number, $a_{0}, a_{1}, \ldots, a_{k+1}, C$ are real constants, and $f\left(a_{0}, a_{1}, \ldots, a_{k+1}\right) \in[0,1)$. Then there exist positive numbers $L$ and $\theta \in(0,1)$ such that

$$
x_{n} \leq L \theta^{n} \quad(n=1,2, \ldots), L=\max _{i=1,2, \ldots, k}\left(x_{i} \theta^{-i}\right) \text {. }
$$

Applying this lemma to the sequence $\left\{\rho\left[x_{n}, x_{n+1}\right]\right\}$, we obtain, according to (3),

$$
\rho\left[x_{n}, x_{n+1}\right] \leq \theta^{n} \max _{i=1, \ldots, k}\left(\rho\left[x_{i}, x_{i+1}\right] \theta^{-i}\right), \quad(n \in N, \theta \in(0,1)) .
$$

Hence, for $n, s \in N$,

$$
\begin{aligned}
\rho\left[x_{n}, x_{n+s}\right] & \leq \sum_{j=1}^{n} \rho\left[x_{n+j-1}, x_{n+j}\right]=\max _{i=1, \ldots, k}\left(\rho\left[x_{i}, x_{i+1}\right] \theta^{-i}\right) \sum_{j=1}^{n} \theta^{n+j-1} \\
& =\max _{i=1, \ldots, k}\left(\rho\left[x_{i}, x_{i+1}\right] \theta^{-i}\right) \theta^{n}(1-\theta)^{-1}, \quad(n \in N, \theta \in(0,1)),
\end{aligned}
$$

which implies that $\left\{x_{n}\right\}$ is a Cauchy sequence. Hence, by the completeness of $X,\left\{x_{n}\right\}$ converges to some point $\xi$ in $X$. For the point $\xi \in X$,

$$
\begin{aligned}
& \rho\left[\xi, T_{n}(\xi)\right] \leq \rho\left[\xi, x_{m+1}\right]+\rho\left[x_{m+1}, T_{n}(\xi)\right]=\rho\left[\xi, x_{m+1}\right]+\rho\left[T_{m+1}\left(x_{m}\right), T_{n}(\xi)\right] \\
& \leq \rho\left[\xi, x_{m+1}\right]+f\left(\alpha_{1} \rho\left[x_{m}, \xi\right], \alpha_{2} \rho\left[x_{m}, x_{m+1}\right], \alpha_{3} \rho\left[\xi, T_{n}(\xi)\right],\right. \\
&\left.\frac{1}{2} \alpha_{4} \rho\left[x_{m}, T_{n}(\xi)\right], \frac{1}{2} \alpha_{5} \rho\left[\xi, x_{m+1}\right]\right) .
\end{aligned}
$$

https://doi.org/10.1017/S0004972700024485 Published online by Cambridge University Press 
Applying this lemma to the sequence $\left\{\rho\left[x_{m+1}, T_{n}(\xi)\right]\right\}$, we obtain, according to (3),

$$
\rho\left[\xi, T_{n}(\xi)\right] \leq \rho\left[\xi, x_{m+1}\right]+L \theta^{m}(m \in N, \theta \in(0,1)) .
$$

Letting $m \rightarrow \infty$, we have $\rho\left[\xi, T_{n}(\xi)\right]=0$; that is, the point $\xi$ is a common fixed point of all $T_{n}$.

To show that $\xi$ is the unique common fixed point of all $T_{n}$, we consider a point $y$ in $X$ such that $T_{n}(y)=y$ for every $n$. Then we have

$$
\begin{aligned}
& \rho[\xi, y]=\rho\left[T_{n}(\xi), T_{n}(y)\right] \leq f\left(\alpha_{1} \rho[\xi, y], \alpha_{2} \rho\left[\xi, T_{n}(\xi)\right], \alpha_{3} \rho\left[y, T_{n}(y)\right],\right. \\
& \left.\frac{1}{2} \alpha_{4} \rho\left|\xi, T_{n}(y)\right|, 3_{2} \alpha_{5} \rho\left|y, T_{n}(\xi)\right|\right) \leq \delta_{0} \rho[\xi, y] \text {. }
\end{aligned}
$$

Hence $\rho[\xi, y]=0$; that is, $\xi=y$. This completes the proof of Theorem 1 .

Now we give an example of a family of maps satisfying the conditions of Theorem 1, for which the conditions of Theorem I do not hold.

EXAMPLE. Let $X=[0,10]$ be the subset of reals with the usual metric and let $\left\{T_{n}\right\}$ be a sequence of functions which maps $X$ into itself, defined as follows:

$$
T_{n}(x)= \begin{cases}0 & , x \in[0,10 / 11) \\ 10 n x(1+11 n)^{-1}, & x \in[10 / 11,10] ; n=0,1,2, \ldots .\end{cases}
$$

Then the conditions of Theorem I of Iséki [2] are not satisfied, since for $x=10 / 11, y=1$; while our conditions of Theorem 1 are satisfied for the mapping

$$
\begin{aligned}
& f\left(t_{1}, \ldots, t_{5}\right) \stackrel{\text { def }}{=}\left(t_{1}+t_{2}+t_{3}\right)^{2}\left(t_{1}+t_{2}+t_{3}-t_{4}\right)^{-1}+t_{5}+t_{6} \\
& \left(t_{1}+t_{2}+t_{3}>2 t_{4}, t_{i} \in R\right)
\end{aligned}
$$

with $\alpha_{1}=\alpha_{2}=1, \alpha_{3}=0, \alpha_{4}=-3, \alpha_{5}=\alpha_{6}=0$. 
2 .

In a recent paper [1], Ćirić proved the following:

THEOREM $C$. Let $F=\left\{T_{\lambda}: \lambda \in I\right\}$ be a family of functions which maps a complete metric space $(X, \rho)$ into itself and let $0 \leq q<1$. If there exists some $T_{\lambda_{0}} \in F$ such that for each $T_{\lambda} \in F\left(\lambda \neq \lambda_{0}\right)$ there are positive integers $i_{\lambda}$ and $j_{\lambda}$ such that

$$
\begin{aligned}
& \rho\left[{ }_{T_{\lambda}}^{i}{ }_{0}{ }_{x},{ }_{T}^{j} \lambda_{\lambda} y\right] \leq q \cdot \max \left\{\rho[x, y], \rho\left[x, T_{\lambda_{0}}^{i} \lambda_{x}\right], \rho\left[y, T_{\lambda}^{j} y\right]\right. \text {, } \\
& \left.\frac{1}{2}\left\{\rho\left[x, T_{\lambda}^{j} \lambda_{y}\right]+\rho\left[y, T_{\lambda_{0}}^{i} \lambda_{x}\right]\right)\right\},
\end{aligned}
$$

holds for all $x, y \in X$, then every $T_{\lambda} \in F$ has a unique fixed point in $X$, which is a unique common fixed point for $F$.

Let $F=\left\{T_{\lambda}: \lambda \in I\right\}$ be a family of functions each of which maps a metric space $(X, \rho)$ into itself. If there exists some $T_{\lambda_{0}} \in F$ such that for each $T_{\lambda} \in F \quad\left(\lambda \neq \lambda_{0}\right)$ there are positive integers $i, j$, and for every $x, y \in X$ there exist real numbers $\alpha_{i}(i=1, \ldots, 5)$ such that

$$
\begin{gathered}
\rho\left[T_{\lambda_{0}}^{i}(x), T_{\lambda}^{j}(y)\right] \leq f\left(\alpha_{1} \rho[x, y], \alpha_{2} \rho\left[x, T_{\lambda_{0}}^{i}(x)\right], \alpha_{3} \rho\left[y, T_{\lambda}^{j}(y)\right],\right. \\
\sin _{2} \rho\left[x, T_{\lambda}^{j}(y)\right], \frac{\left.z_{2} \alpha_{5} \rho\left[y, T_{\lambda_{0}}^{i}(x)\right]\right),}{} \\
\sup \left\{f\left(\alpha_{1}, \alpha_{2}, \alpha_{3}, \alpha_{4}, \alpha_{5}\right): x, y \in X\right\}=\delta \in[0,1),
\end{gathered}
$$

where the mapping $f: R^{5} \rightarrow R$ is increasing, semihomogeneous and $g(x)=f\left(\alpha_{1} x, \ldots, \alpha_{5} x^{5}\right)$ continuous in the point $x=1$, then mappings $T_{\lambda}$ of this type will be referred to as having property $(f)$ over $X$.

THEOREM 2. Let $F=\left\{T_{\lambda}: \lambda \in I\right\}$ be a family of functions which maps a complete metric space $(X, \rho)$ into itself, and let $T_{\lambda} \in F$ have 
property $(f)$ over $X$. Then every $T_{\lambda} \in F$ has a fixed point in $X$, and it is unique when $\delta_{0}<1$, and it is then the urique common fixed point for $F$.

Proof. Let $T_{\lambda} \in F$ be arbitrary. For arbitrary $x \in X$ let us consider a sequence

(5) $x_{0}=x, x_{1}=T_{\lambda_{0}}^{i} x_{0}, x_{2}=T_{\lambda}^{j} x_{1}, \ldots, x_{2 n-1}=T_{\lambda_{0}}^{i} x_{2 n-2}$,

$$
x_{2 n}=T^{j} x_{2 n-1}^{j}, \cdots \text {. }
$$

By assertion (4) we have

$$
\begin{aligned}
& \rho\left[x_{2 n}, x_{2 n+1}\right]=\rho\left[T^{j} x_{2 n-1}, T_{\lambda_{0}}^{i} x_{2 n}\right] \leq \\
& \quad \leq f\left(\alpha_{1} \rho\left[x_{2 n-1}, x_{2 n}\right], \alpha_{2} \rho\left[x_{2 n-1}, T_{\lambda}^{j} x_{2 n-1}\right], \alpha_{3} \rho\left[x_{2 n}, T_{\lambda_{0}}^{i} x_{2 n}\right]\right. \\
& \left.\quad \frac{1}{2} \alpha_{4} \rho\left[x_{2 n-1}, T_{\lambda_{0}}^{i} x_{2 n}\right], \frac{1}{2} \alpha_{5} \rho\left[x_{2 n}, T_{\lambda}^{j} x_{2 n-1}\right]\right)=f\left(\alpha_{1} \rho\left[x_{2 n-1}, x_{2 n}\right],\right. \\
& \left.\quad \alpha_{2} \rho\left[x_{2 n-1}, x_{2 n}\right], \alpha_{3} \rho\left[x_{2 n}, x_{2 n+1}\right], \frac{1}{2} \alpha_{4} \rho\left[x_{2 n-1}, x_{2 n+1}\right], \frac{1}{2} \alpha_{5} \rho\left[x_{2 n}, x_{2 n}\right]\right) .
\end{aligned}
$$

Then by a routine calculation one can show that (5), from the lemma, is a Cauchy sequence. Using completeness of $X$ we have that $\underset{n}{\lim } x_{n}=\xi$, for some $\xi \in X$. By assertion (4),

$$
\begin{aligned}
\rho\left[\xi, T_{\lambda_{0}}^{i}(\xi)\right] \leq \rho\left[\xi, x_{2 n}\right]+\rho\left[x_{2 n}, T_{\lambda_{0}}^{i}(\xi)\right] \leq \rho\left[\xi, x_{2 n}\right]+f\left(\alpha_{1} \rho\left[x_{2 n-1}, \xi\right],\right. \\
\left.\alpha_{2} \rho\left[x_{2 n-1}, x_{2 n}\right], \alpha_{3} \rho\left[\xi, T_{\lambda_{0}}^{i}(\xi)\right], \frac{3}{2} \alpha_{4} \rho\left[x_{2 n-1}, T_{\lambda_{0}}^{i}(\xi)\right], \frac{1}{2} \alpha_{5} \rho\left[\xi, x_{2 n}\right]\right) .
\end{aligned}
$$

Applying the lemma to the sequence $\left\{\rho\left[x_{2 n}, T_{\lambda_{0}}^{i}(\xi)\right]\right\}$, we obtain according to $(3)$,

$$
\rho\left[\xi, T_{\lambda_{0}}^{i}(\xi)\right] \leq \rho\left[\xi, x_{2 n}\right]+L \theta^{n} \quad(n \in N, \theta \in(0,1)) .
$$

Letting $n \rightarrow \infty$, then we have $\rho\left[\xi, T_{\lambda_{0}}^{i}(\xi)\right]=0$; that is, the point $\xi$ is a common fixed point of $T_{\lambda_{0}}^{i}$, and it is unique. 
By Theorem 1 and assertion (4), $\xi$ is a unique fixed point of $T_{\lambda_{0}}^{i}$ and $T_{\lambda}^{j}$. Since $T_{\lambda_{0}}^{i}\left(T_{\lambda_{0}} \xi\right)=T_{\lambda_{0}}\left(T_{\lambda_{0}}^{i} \xi\right)=T_{\lambda_{0}} \xi, T_{\lambda_{0}} \xi$ is also a fixed point of $T_{\lambda_{0}}^{i}$, and therefore $T_{\lambda_{0}} \xi=\xi$. Similarly it follows that $T_{\lambda} \xi=\xi$. So we have proved that $\xi$ is a unique fixed point of ${ }^{T} \lambda_{0}$ and $T_{\lambda}$

Now we shall show that $\xi$ is a unique common fixed point for $F$. Let $T_{\lambda_{1}} \in F \quad\left(\lambda_{0} \neq \lambda_{1} \neq \lambda\right)$, be arbitrary. Since $\xi=T_{\lambda_{0}} \xi$ implies $\xi=T_{\lambda_{0}}^{i} \xi$, by (4) and Theorem I, $\xi$ is a unique fixed point of $T_{\lambda}^{j}$. This implies that $\xi$ is a unique fixed point of $T_{\lambda_{I}}$. This completes the proof of Theorem 2.

THEOREM 3. Let $X$ be a metric space, and let $\left\{T_{n}\right\}$ be a sequence of mappings wrich map $X$ into itself. Let $T: X \rightarrow X$ be an $f$-contraction and let $X$ be T-orbitally complete, and $\delta_{0}<1$. If each $T_{n}$ has at least one fixed point $u_{n}$, and if we suppose that $\left\{T_{n}\right\}$ converges uniformly to $T$, then the sequence $\left\{u_{n}\right\}$ converges to the unique fixed point $u$ of $T$.

Proof. By Theorem $T, T: X \rightarrow X$ has a unique fixed point $u$. We have

$$
\begin{aligned}
& \rho\left[u, u_{n}\right]=\rho\left[T u, T_{n}\left(u_{n}\right)\right] \leq \rho\left[T u, T\left(u_{n}\right)\right]+\rho\left[T\left(u_{n}\right), T_{n}\left(u_{n}\right)\right] \leq \\
& \leq f\left(\alpha_{1} \rho\left[u, u_{n}\right], \alpha_{2} \cdot 0, \alpha_{3} \rho\left[u_{n}, T\left(u_{n}\right)\right], z_{2} \alpha_{4} \rho\left[u, T\left(u_{n}\right)\right],\right. \\
& \left.\frac{1}{2} \alpha_{5} \rho\left[u_{n}, u\right]\right)+\rho\left[T\left(u_{n}\right), T_{n}\left(u_{n}\right)\right] \text {. }
\end{aligned}
$$

Applying the lemma to the sequence $\left\{\rho\left[u, u_{n}\right]\right\}$ we obtain, according to (3),

$$
\rho\left[u, u_{n}\right] \leq L \theta^{n}+\rho\left[T\left(u_{n}\right), T_{n}\left(u_{n}\right)\right] \quad(n \in N, \theta \in(0,1)) .
$$

Since $\left\{T_{n}\right\}$ converges uniformly to $T$, 


$$
\rho\left[u_{n}, T\left(u_{n}\right)\right]=\rho\left[T_{n}\left(u_{n}\right), T\left(u_{n}\right)\right] \rightarrow 0 \quad(n \rightarrow \infty)
$$

and we have that $\lim \rho\left[u, u_{n}\right]=0$, which completes the proof.

\section{References}

[1] Ljubomir B. Ćirić, "On a family of contractive maps and fixed points", Publ. Inst. Math. (Beograd) (NS) 17 (31) (1974), 45-51.

[2] Kiyoshi Iséki, "On common fixed points of mappings", Bulz. Austraz. Math. Soc. 10 (1974), 365-370.

[3] Baidyanath Ray, "Some results on fixed points and their continuity", Cozzoq. Math. 27 (1973), 41-48.

[4] Milan R. Tasković, "Monotonic mappings on ordered sets, a class of inequalities with finite differences and fixed points", Publ. Inst. Math. (Beograd) (NS) 17 (31) (1974), 163-172.

[5] Milan R. Tasković. "Eine Abbilđung von B-Typus", Publ. Inst. Math. (Beograd) (NS) 17 (32) (1974), 197-206.

[6] Milan R. Tasković, "A generalization of Banach's contraction principle", submitted.

Prirodno-metemtički facultet,

Beograd,

Yugoslavia. 